\title{
A PROPERTY OF INDECOMPOSABLE CONNECTED SETS
}

\section{MARY ELLEN RUDIN}

Introduction. 'Two nonempty subsets of a topological space are said to be separated if neither intersects the closure of the other. A set is connected if it is not the union of two separated sets. A connected set $I$ is indecomposable if it is not the union of two connected sets, neither of which is dense in $I$.

In [1], Swingle raised the following question: does there exist, in the plane, an indecomposable connected set $I$, such that the set $I \cup\{p\}$ fails to be indecomposable for some limit point $p$ of $I$ ?

The purpose of this paper is to prove that the answer is negative. It is interesting to note that the plane plays an essential role here as the embedding space: if the plane is replaced by Euclidean 3-space in Swingle's question, the answer turns out to be affirmative. The construction of such an example is rather complicated, and is not included in this paper.

Notation. A component of a set is a maximal connected subset.

If $X$ and $Y$ are sets, $X-Y$ denotes the set of all elements of $X$ which are not elements of $Y$ (whether $Y$ is a subset of $X$ or not).

The boundary of a set $X$ will be denoted by $\partial X$, and $\bar{X}$ will denote the closure of $X$.

Theorem. Suppose I is an indecomposable connected subset of the plane and $p$ is a limit point of $I$. Then $I \cup\{p\}$ is also an indecomposable connected set.

Proof. We will begin with three rather basic and fairly evident lemmas. Then we will prove Lemma 4, whose purpose may at first glance be obscure to the reader. The proof of Lemma 4 depends on the fact that if there are three arcs in the plane which do not contain the point $p$ and which have only their end-points in common, then one of these arcs is separated from $p$ in the plane by the union of the other two. Then, assuming that the theorem is false, Steps 1 and 2 of the proof will describe the construction of some rather complicated sets. In Step 3, a set $J$ will be defined and, using Lemma 4, it will be proved that $J \neq 0$. In Step 4 , an argument analogous to the proof of Lemma 4 will prove that $J=0$, and this contradiction implies the theorem.

Suppose $X$ is a set in the plane. We will say that $X$ has property $\lambda$ if neither $X$ nor $I-X$ is dense in $I$. We will use $\partial_{I} X$ to denote the boundary of $X$ with respect to $I$; that is, $\partial_{I} X=I \cap \bar{A} \cap \bar{B}$

Received by the editors January 28, 1957. 
where $A=I \cap X$ and $B=I-X$. It should be noticed that, since $I$ is connected, if $A \neq 0$ and $B \neq 0$, then $\partial_{I} X \neq 0$.

Lemma 1. If a set $E$ is a subset of I having property $\lambda$, then $E$ is not connected.

Proof. Assume $E$ is connected. Since $\bar{E}$ has property $\lambda$ and $I$ is indecomposable, $I-\bar{E}$ cannot be connected. So $I-\bar{E}$ is the union of two separated sets $A_{1}$ and $A_{2}$. If $\bar{E} \cup A_{1}$ were the union of two separated sets $B_{1}$ and $B_{2}$ with $B_{2} \subset A_{1}$, then the sets $B_{2}$ and $B_{1} \cup A_{2}$ would be separated; but $I=B_{1} \cup B_{2} \cup A_{2}$, and $I$ is connected. This contradiction shows that $\bar{E} \cup A_{1}$ is connected. Similarly $\bar{E} \cup A_{2}$ is connected. Since $A_{1}$ and $A_{2}$ are open with respect to $I$, neither $\bar{E} \cup A_{1}$ or $\bar{E} \cup A_{2}$ is dense in $I$. Since $I$ is indecomposable this is impossible and our lemma is proved.

Lemma 2. If $X$ has property $\lambda$, there are disjoint open sets $Q_{i}(X)$ $(i=1,2,3)$ whose union contains $I-X$, such that $Q_{i}(X)$ is open in the plane, $p \notin Q_{i}(X)$, and $Q_{i}(X)$ intersects $I-X$.

Proof. It is clearly sufficient to prove that $I-X$ is the union of three separated sets.

In order to prove this observe that $I-X$ has property $\lambda$. By Lemma 1, then $I-X=A_{1} \cup A_{2}$ with $A_{1}$ and $A_{2}$ separated. One of the sets $A_{1}$ or $A_{2}$ has property $\lambda$, say $A_{1}$. Then $A_{1}=B_{1} \cup B_{2}$ with $B_{1}$ and $B_{2}$ separated. Hence $I-X=A_{2} \cup B_{1} \cup B_{2}$ and $A_{2}, B_{1}$ and $B_{2}$ are separated.

Lemma 3. Suppose $X \subset I, H \subset X \cup\{p\}, H$ is connected and $X$ is the union of two separated sets $A$ and $B$. Then $E=(A \cap H) \cup\{p\}$ is connected.

Proof. If $A \cap H=0$ then the theorem is trivially correct. If $A \cap H \neq 0$, suppose $E$ is the union of two separated sets $E_{1}$ and $E_{2}$ and $p \in E_{1}$. Then $E_{2}$ and $B$ are separated. But $H=E_{1} \cup E_{2} \cup(B \cap H)$, and $E_{2}$ is separated from $E_{1} \cup(B \cap H)$. Since $H$ is connected, this is impossible.

Lemma 4. (1) Suppose $U$ and $V$ are disjoint connected open sets in the plane having property $\lambda$ and not containing $p$.

(2) Suppose $X=(I \cup\{p\})-(U \cup V)$ is connected.

Then, for some $i, Q_{i}(U) \cap \bar{V} \cap I=0 .\left(Q_{i}(U)\right.$ is defined in accordance with Lemma 2, and $i$ is 1, 2, or 3.)

Proof. Assume that, for each $i, I \cap Q_{i}(U) \cap \bar{V} \neq 0$. If $I \cap \bar{U} \cap \bar{V}$ $\cap Q_{i}(U)$ exists let $x_{i}$ be a point of this set. Otherwise for each $i$, let 
$T_{i}$ denote a component of $\left[Q_{i}(U)-\bar{U}\right]$ intersecting $\bar{V} \cap I$. Since $I \cap \partial T_{i} \neq 0$ and $I \cap \partial T_{i} \subset \partial U$, we can choose, for each $i$, a point $x_{i} \in I \cap \partial T_{i} \cap \partial U$. Also choose $x \in U$ and $y \in V$.

Letting $Q_{i}$ be the component of $Q_{i}(U)$ which contains $x_{i}$, it is clear that $U \cap Q_{i} \neq 0$, and $V \cap Q_{i} \neq 0$. One can therefore construct arcs $L_{i}(i=1,2,3)$ with the following properties:

(a) $L_{i}$ has $x$ and $y$ as end-points.

(b) $L_{i} \subset U \cup Q_{i} \cup V$, and $x_{i} \in L_{i}$.

(c) If $i \neq j$, then $L_{i} \cap L_{j}=\{x\} \cup\{y\}$.

The plane is separated by $L_{1} \cup L_{2} \cup L_{3}$ in such a way that $p$ is separated from one of these arcs by the other two. Say, $p$ is separated from $L_{1}$ by $L_{2} \cup L_{3}$. Then consider the set $A=Q_{1}(U)-(V \cup U)$. By Lemma 3 and assumption (2), $A \cup\{p\}$ is connected. But $x_{1} \in A$ and $A \cup\{p\}$ does not intersect $L_{2} \cup L_{3}$. This contradicts the connectedness of $A \cup\{p\}$, and proves the lemma.

We now turn to the proof of the theorem.

SteP 1. On the assumption that the theorem is false, we would like to show that there are disjoint connected open sets $V$ and $W$ in the plane intersecting I such that

$$
H=(I \cup\{p\})-(V \cup W) \text { is connected. }
$$

It is obvious that $I \cup\{p\}$ is connected; thus if the theorem is false, $I \cup\{p\}$ is the union of two connected sets $H_{1}$ and $H_{2}$ neither of which is dense in $I \cup\{p\}$ such that $p \in H_{1}$.

Since $I-\bar{H}_{1}$ has property $\lambda$, Lemma 1 shows that $I-\bar{H}_{1}$ is the union of two separated sets $K_{1}$ and $K_{2}$. For $i=1$, 2, let $G_{i}$ be open sets such that $G_{i} \supset K_{i}, G_{i} \cap H_{1}=0, G_{1} \cap G_{2}=0$. Suppose $V$ and $W$ are components of $G_{1}$ and $G_{2}$, respectively, intersecting $I$. In order to show that $H$ is then connected, let us suppose that $H=A \cup B$ with $A$ and $B$ separated and $p \in A$. Since $H_{1} \subset H$ and $H_{1}$ is connected, $H_{1} \subset A$. But $B$ is closed with respect to $I$ and $\partial_{I} B \subset \bar{H}_{1}$. Hence $A$ and $B$ are not separated and this contradiction shows that $H$ is connected.

Step 2. We now define a well ordered sequence of plane sets $F_{\alpha}$ : Put $F_{1}=W$. If $\alpha$ is an ordinal and $F_{\gamma}$ has been defined for $1 \leqq \gamma<\alpha$, put $S_{\alpha}=U_{\gamma<\alpha} F_{\gamma}$. If there exist separated sets $A$ and $B$ such that $I-S_{\alpha}=A \cup B$ and $A \supset \bar{V} \cap I$, then choose one such pair $A, B$ and put $F_{\alpha}=B$. Let $\beta$ be the first ordinal such that $I-S_{\beta}$ cannot be decomposed in the above manner; the well ordered sequence in question consists of the sets $F_{\alpha}$ with $1 \leqq \alpha<\beta$.

For $\alpha \leqq \beta$, let $G_{\alpha}=S_{\alpha}-W$; and $G=G_{\beta}$.

Let $M$ and $N$ be disjoint open sets not containing $p$ such that $M \supset S_{\beta}$ and $N \supset\left(I-\bar{S}_{\beta}\right) \cup V$. Let $L$ denote the component of $N$ con- 
taining $V$. For $\alpha<\beta$, let $M_{\alpha}$ be an open subset of $M$ such that $M_{\alpha} \supset G_{\alpha}$ and $\left(I \cap \bar{M}_{\alpha}\right)-W=\bar{G}_{\alpha} \cap I$.

We would like to point out the following facts.

1. If $U \subset S_{\beta}, Y \subset I-U, Y \cap \bar{V}=0$, and $Y$ and $(I-U)-Y$ are separated, then $Y \subset S_{\beta}$. If $Y-S_{\beta} \neq 0$, then $Y-S_{\beta}$ and $\left(I-S_{\beta}\right)-Y$ are separated, which contradicts the definition of $\beta$.

2. For $\alpha \leqq \beta, G_{\alpha} \cup\{p\}$ is connected. This can be seen by supposing $\gamma$ is the smallest ordinal such that $G_{\gamma} \cup\{p\}$ is not connected. Clearly $\gamma$ is not a limit ordinal since in this case $G_{\gamma}=\bigcup_{\delta<\gamma} G_{\delta}$. Also $\gamma \neq 1$, since $G_{1}=0$. Hence $G_{\gamma-1} \cup\{p\}$ is connected and $G_{\gamma} \cup\{p\}=G_{\gamma-1} \cup\{p\}$ $\cup F_{\gamma-1}$. Therefore, if $G_{\gamma} \cup\{p\}=A \cup B$ with $A$ and $B$ separated, suppose $B \supset G_{\gamma-1} \cup\{p\}$. Then $A \subset F_{\gamma-1}$ and $A$ and $I-S_{\gamma}$ are separated, by the definition of $F_{\gamma-1}$. Since $I-W \subset\left(I-S_{\gamma}\right) \cup A \cup B, A$ and $(I-W)-A$ are separated. And since $(H-\{p\}) \subset(I-W), A$ and $(H-\{p\})-A$ are separated. Hence, by Lemma $3, A \cup\{p\}$ is connected. But then $A$ and $B$ are not separated and this contradiction shows that $G_{\gamma} \cup\{p\}$ is connected.

STEP 3. We will now consider $Q_{i}(L), i=1,2,3,[L$ is defined in paragraph 3 of Step 2 and $Q_{i}(L)$ is defined in Lemma 2.]

Let $J=Q_{i}(L) \cap I \cap(\bar{G}-G)$. In this step we will show that $J \neq 0$.

Suppose $J=0$. Let $U$ denote a component of $Q_{i}(L) \cap M$. In order to use Lemma 4 we would like to show that $X=(I \cup\{p\})-(U \cup V)$ is connected.

First let us show that $H \cap X$ is connected. Since $J=0, U \cap G$, and $I-U$ are separated; hence $(X \cap H)-\{p\}=A$ and $B=(U \cap G)$ are separated. But then $H-\{p\}=A \cup B$, so by Lemma 3, $H \cap X$ $=A \cup\{p\}$ is connected.

Suppose $X=D \cup E, D$ and $E$ are separated, and $H \cap X \subset D$. But $\partial_{I} E \subset \partial_{I} X \subset(I \cap(\partial U \cup \partial V)) \subset H$ since $I \cap \partial U \cap W=0$ and $U \cap V=0$. Therefore $\partial_{I} E \subset H$ and since $E$ is closed relative to $I$ this contradicts the separation of $D$ and $E$. Hence $X$ is connected.

We can now apply Lemma 4 and conclude that, for some $j$, $Q_{j}(U) \cap \bar{V} \cap I=0$.

Let $Y=\left(Q_{j}(U) \cap I\right)-U$. Then $U \subset S_{\beta}, Y \subset I-U, Y \cap \bar{V}=0$ and $Y$ and $(I-U)-Y$ are separated. Therefore by 1 of Step 2, $Y \subset S_{\beta}$. Choose $y \in \partial_{I} Y$. There is an open disk lying in $Q_{i}(L) \cap M$ containing $y$ and, since $y \in \partial U$, this disk intersects $U$. But since $U$ is a component of $Q_{i}(L) \cap M$, the disk is in $U$. This contradiction shows that $J \neq 0$.

Step 4. We will now reach a contradiction by a proof somewhat similar to that of Lemma 4.

For $i=1,2,3$, let $\beta_{i}$ denote the smallest ordinal such that 
$J \cap \bar{G}_{\beta_{i}} \neq 0$. Observe that $\beta_{i}$ is a limit ordinal since for nonlimit ordinals $\alpha, G_{\alpha}=G_{\alpha-1} \cup F_{\alpha-1}$ and $\bar{F}_{\alpha-1} \cap(\bar{G}-G)=0$. Choose $y_{i} \in J \cap \bar{G}_{\beta_{i}}$.

Suppose $D_{i n}$ is an open disk containing $y_{i}$ of radius $1 / n$. Then, since $\beta_{i}$ is a limit ordinal, there is an ordinal $\alpha_{i n}<\beta_{i}$ such that either

(a) there is a point $W_{i n} \in D_{i n} \cap G_{\alpha_{i n}} \cap \partial W$ or

(b) there is a component $R_{i n}=R$ of $\left(M_{\alpha_{i n}} \cap Q_{i}(L)\right)-\bar{W}$ such that $R \cap G_{\alpha_{i n}} \cap D_{i n} \neq 0$.

In case (b) $\partial_{I} R \cap \partial W \neq 0$. This can be shown by letting $\gamma$ be the smallest ordinal such that $F_{\gamma} \cap R \neq 0$. Then

$$
G_{\gamma} \cap R=0 .
$$

If $r \in \partial_{I}\left(F_{\gamma} \cap R\right)$ then $r \in \partial W$ for if $\left.r \notin \bar{W}, r \in M_{\alpha_{i n}} \cap Q_{i}{ }^{\prime} L\right)$ and is hence an interior point of $R$. Since $\partial_{I} F_{\gamma} \subset \bar{W} \cup \bar{G}_{\gamma}$, if $r \notin \bar{W}, r \in \bar{G}_{\gamma}$ and $r \in R$. Since $R$ is open this contradicts the fact that $G_{\gamma} \cap R=0$. Hence in case (b) there is a point $w_{i n} \in \partial_{I} R \cap \partial W$.

The following fact about the plane will be used without proof in the following two paragraphs. If $W$ is a connected open set, $w$ a point of $W, Z$ a finite subset of $\partial W$, and $\epsilon$ is a positive number, then for each point $z$ of $Z$ there are $\operatorname{arcs} L(z)$ from $w$ to $z$ and open disks $D(z)$ containing $z$ such that,

(1) if $x \neq z, L(z) \cap L(x)=\{w\}$,

(2) if $x \neq z, D(z) \cap(L(x) \cup D(x))=0$,

(3) $L(z) \subset W \cup D(z)$,

(4) the diameter of $D(z)$ is less than $\epsilon$.

For $i=1,2,3$ let $w_{i}$ be one limit point of the sequence $w_{i 1}, w_{i 2}$, $w_{i 3}, \cdots$. [If necessary, embed the plane in a bigger plane.] Choose a point $w$ of $W$ and let $Z$ be the set consisting of $w_{1}, w_{2}, w_{3}$ and those of the points $y_{1}, y_{2}$, and $y_{3}$ which are in $\partial W$. Then for each $z$ in $Z$ there are $\operatorname{arcs} L(z)$ from $w$ to $z$ and open disks $D(z)$ containing $z$ such that (1), (2), and (3) of the preceding paragraph hold and (4) if $z=y_{i}$ for some $i$, then $D(z) \subset Q_{i}(L)$. For $y_{i} \notin \partial W$, let $D\left(y_{i}\right)$ be a disk such that the closures of $W$ and $D\left(y_{i}\right)$ are disjoint and $D\left(y_{i}\right) \subset Q_{i}(L)$.

For $i=1,2,3$ choose an integer $m$ such that $w_{i m} \in D\left(w_{i}\right)$ and $D_{i m} \subset D\left(y_{i}\right)$; let $w^{i}$ denote $w_{i m}, \alpha_{i}$ denote $\alpha_{i m}$, and $x_{i}$ denote (a) $w^{i}$ if $w^{i} \in D\left(y_{i}\right)$ or (b) a point of $R_{i n} \cap D\left(y_{i}\right)$, if $w^{i} \in D\left(y_{i}\right)$. As before, for $i=1,2,3$ there are arcs $L^{i}$ from $w$ to $w^{i}$ and open disks $D^{i}$ containing $w^{i}$, such that (1), (2) and (3) above follow and (3) $D^{i} \subset\left(M_{\alpha_{i}} \cap Q_{i}(L)\right)$ and for $i \neq j, D^{i} \cap D\left(y_{j}\right)=0$.

There is an open set $S$ such that $S \supset\left(I-S_{\beta}\right) \cup V, S \cap\left(\left(M_{\alpha_{i}} \cap Q_{i}(L)\right.\right.$ $\left.\cup L_{1}\right)=0$ for all $i$, and $p \notin S$. Such a set clearly exists since $L^{i}$ is a closed subset of $\left(M_{\alpha_{i}} \cap Q_{i}(L)\right) \cup W ;\left(\left(I-S_{\beta}\right) \cup V\right) \cap\left(M_{\alpha_{i}} \cup W\right)=0$ for all $i$; and, for $x \in \partial\left(\left(I-S_{\beta}\right)-V\right), x \in Q_{i}(L)-W$ for some $i$ so, for $j \neq i, x$ and 
$Q_{j}(L)$ are separated and, by the definition of $\beta_{i}$ and the fact that $\alpha_{i}<\beta_{i}$, we have that $x \notin \bar{M}_{\alpha_{i}}$.

Let $T$ be the component of $S$ containing $V$. Then $T \supset I-S_{\beta}$ for if $A=(S-T) \cap\left(I-S_{\beta}\right) \neq 0$ then $A$ and $T \cap\left(I-S_{\beta}\right)$ are separated since they are in different components of the open set $S$. Since $A \cap V=0$, this contradicts the definition of $S_{\beta}$. Choose $t \in T$.

Therefore, as in the proof of Lemma 4 , there are arcs $L_{i}(i=1,2,3)$ having the following properties:

(a) $L_{i}$ has $w$ and $t$ as its end points,

(b) $L_{i} \subset\left(W \cup\left(M_{\alpha_{i}} \cap Q_{i}(L)\right) \cup D\left(y_{i}\right) \cup T\right), x_{i} \in L_{i}$,

(c) if $i \neq j$, then $L_{i} \cap L_{j}=\{w\} \cup\{t\}$.

Using Lemma 3 and fact (2) of Step (2) we can see that $\left(G_{\alpha_{i}} \cap Q_{i}(L)\right)$ $\cup\{p\}$ is connected since $G_{\alpha_{i}} \subset\left(Q_{1}(L) \cup Q_{2}(L) \cup Q_{3}(L)\right)$ and $Q_{1}(L)$, $Q_{2}(L)$, and $Q_{3}(L)$ are separated.

The plane is separated by $L_{1} \cup L_{2} \cup L_{3}$ in such a way that $p$ is separated from one of these arcs by the other two. Say $p$ is separated from $L_{1}$ by $L_{2} \cup L_{3}$. But since $x_{1} \in L_{1} \cap G_{\alpha_{1}} \cap Q_{1}(L)$ and since $\left(L_{2} \cup L_{3}\right)$ $\cap\left(\left(G_{\alpha_{1}} \cap Q_{1}(L)\right) \cup\{p\}\right)=0$, this contradicts the connectedness of $\left(G_{\alpha_{1}} \cap Q_{1}(L) \cup\{p\}\right)$; and proves our theorem.

\section{REFERENCE}

1. Paul M. Swingle, The closure of types of connected sets, Proc. Amer. Math. Soc. vol. 2 (1951) pp. 178-185.

UNIVERSITY OF ROCHESTER 